\title{
Coupled Heat Transfer Analysis in Regeneratively Cooled Thrust Chambers
}

\author{
Barbara Betti*, Marco Pizzarelli ${ }^{\dagger}$, Francesco Nasuti ${ }^{\ddagger}$ \\ University of Rome "La Sapienza", Italy
}

\begin{abstract}
A computational procedure able to describe the coupled hot-gas/wall/coolant environment that occurs in most liquid rocket engines and to provide a quick and reliable prediction of thrust-chamber wall temperature and heat flux as well as coolant-flow characteristics, like pressure drop and temperature gain in the regenerative circuit is presented and demonstrated. The coupled analysis is performed by means of an accurate CFD solver of the Reynolds-Averaged Navier-Stokes equations for the hot-gas flow and a simplified quasi-2D approach, which widely relies on semi-empirical relations, to study the problem of coolant flow and wall structure heat transfer in the cooling channels. Coupled computations of the Space Shuttle Main Engine Main Combustion Chamber are performed and compared with available literature data. Results show a reasonable agreement in terms of coolant pressure drop and temperature gain with nominal data, whereas the computed wall temperature peak is quite closer to hot-firing data than to the nominal value.
\end{abstract}

\section{Nomenclature}

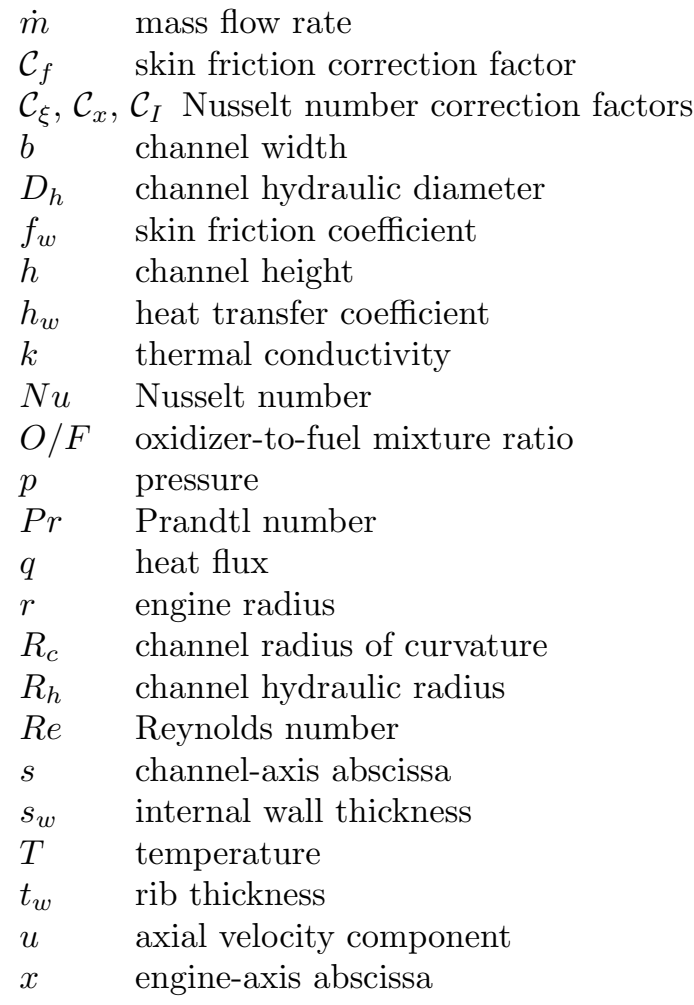

*PhD Student. email: barbara.betti@uniroma1.it

${ }^{\dagger}$ Research Fellow. email: marco.pizzarelli@uniroma1.it

$¥$ Associate Professor. email: francesco.nasuti@uniroma1.it

$\S$ Dipartimento di Ingegneria Meccanica e Aerospaziale, Via Eudossiana 18, 00184 Roma 
$y^{+} \quad$ dimensionless wall distance

\begin{tabular}{ll}
\multicolumn{2}{l}{ Subscripts } \\
0 & total \\
$a w$ & adiabatic wall \\
$c$ & chamber \\
$f$ & film \\
$h g$ & hot-gas side \\
$t$ & turbulent \\
$w$ & wall \\
Symbols \\
$\rho$ & density \\
$\varepsilon$ & wall roughness \\
$\xi$ & rough-to-smooth friction ratio
\end{tabular}

\section{Introduction}

In order to keep the temperatures of the thrust chamber walls within their allowed limits, an intense cooling is necessary, which, in case of regenerative cooling, is achieved by flowing a coolant into suitable channels surrounding the thrust chamber. Due to the strongly coupled nature of the heat transfer problem, coolant and hot gas side cannot be studied independently. For this reason, many studies have been published in the literature to solve the coupled heat transfer problem of hot gas, chamber wall and coolant in thrust chambers.

The most common approaches rely on the one-dimensional analysis with Nusselt-type empirical relations for both hot-gas and coolant side. ${ }^{1-7}$ To overcome the simplification arising from one-dimensional modeling of hot-gas expansion, efforts have been made to describe the hot-gas flow-field by means of multi-dimensional CFD solvers, ${ }^{8-12}$ while maintaining semi-empirical modeling for the coolant flow. Recently, coupled CFD analysis for the whole regeneratively-cooled thrust chamber has been performed. ${ }^{13,14}$

All of these approaches have their well-defined objective that in some cases lead to fast and simplified methods and in other cases to heavy computations which are not suited to engine design phase.

Objective of the present work is to present and discuss merits and limitations of a computational procedure able to describe the coupled hot-gas/wall/coolant environment that occurs in most liquid rocket engines and to provide a quick and reliable prediction of thrust-chamber wall temperature and heat flux as well as coolant-flow characteristics, like pressure drop and temperature gain in the regenerative circuit. In the present coupled approach, the hot-gas side flow-field and heat transfer are computed by means of a CFD solver ${ }^{15}$ while the coolant flow and the wall structure heat transfer are computed by means of a simplified quasi-2D approach, ${ }^{7}$ which widely relies on semi-empirical relations. The quasi-2D model is able to take into account for the real fluid nature of the coolant and the thermal stratification inside the channels which cannot be neglected in actual cooling channels. Performed coupled computations of the Space Shuttle Main Engine (SSME) Main Combustion Chamber are analyzed in detail and compared with available literature data.

\section{Modeling}

A comprehensive thermal model for a regeneratively cooled thrust chamber must account for convection from hot-gas, conduction within the wall, and convection to the coolant. In fact, heat transfer can be described as the heat flux between two fluids, separated by a solid wall. In its simplest form regenerative cooling can be modeled as a steady heat flux from a hot gas through a solid wall to a cold fluid. This problem can be divided into three sub-problems, which are defined as follows:

Hot gas The turbulent flow of a mixture of gases in a rocket engine, including combustion chamber and convergent-divergent nozzle.

Wall The heat conduction through the wall of the rocket engine between the hot gas and the coolant. 
Coolant The turbulent flow of the coolant in the channels surrounding the rocket engine.

These sub-problems are coupled by the two steady-state balances of three heat fluxes: from hot-gas to wall; through the wall; and from wall to coolant.

\section{A. Hot gas: CFD solver}

A Reynolds-Averaged Navier-Stokes (RANS) approach is used to obtain CFD solutions of the hot-gas flowfield and heat transfer in the thrust chamber. In particular, combustion is not simulated, but a flow of combustion products in chemical equilibrium at the chamber temperature and pressure is injected using a "full-inlet" approach. ${ }^{16}$ The numerical solutions are carried out by means of an in-house 3D multi-block finite volume RANS equations solver able to treat multi-component mixtures of thermally perfect gases. Multicomponent diffusion has been validated ${ }^{17}$ reproducing experimental test cases retrieved in open literature. Turbulence is described by means of the Spalart-Allmaras one equation model ${ }^{18}$ and a constant turbulent Schmidt number is adopted to model turbulent diffusivity. Wall heat flux evaluation has been validated by reproducing a benchmark test case involving a supersonic nozzle operated with air. ${ }^{16}$

\section{B. Coolant and wall: quasi-2D solver}

A simplified quasi-2D approach ${ }^{7}$ is used to study the coupled problem of coolant flow and wall structure heat transfer in rectangular cooling channels of liquid rocket engine thrust chambers. The cooling channel flow model is developed by using the steady-state conservation laws of mass, momentum, and energy, taking into account the effects of heat transfer and friction. In particular, the mass and momentum governing equations are written in a one-dimensional form, whereas a simplified arrangement of the $2 \mathrm{D}$ energy equation is considered. In fact, the fluid energy equation can be reduced to a $2 \mathrm{D}$ balance in the stream-wise direction and radial direction. For that reason this flow model is referred to as quasi-2D. Moreover, to accurately describe the wall heat flux, also the axial component of the fluid velocity and the wall temperature $T_{w}$ are assumed to vary along the radial direction. Likewise to $1 \mathrm{D}$ analysis the transverse velocity components are neglected because they are much smaller than the axial component. According to the above hypotheses, the fluid pressure only depends on the stream-wise coordinate position, whereas the fluid temperature and axial velocity and wall temperature are left to vary also in radial direction. The model, aiming to the study of any fluid evolving through cooling channels, considers a generic equation of state, and thus the coolant fluid can be either a compressible gas, or a supercritical fluid or a liquid. The turbulent thermal conductivity, fluid skin friction and coolant-wall heat transfer coefficients are evaluated by semi-empirical relations provided in the literature. In this study, the turbulent thermal conductivity $k_{t}$ in the radial direction is modeled according to: ${ }^{19}$

$$
\frac{k_{t}}{k}=0.008 \cdot R e^{0.9}
$$

where $k$ is the fluid thermal conductivity and $R e$ is the Reynolds number. The coolant friction is composed of two terms: the first, $f_{w}^{0}$, expresses the skin friction for the case of constant properties flow (which grossly represents the adiabatic flow) in rough tubes; the second, $\mathcal{C}_{f}$, is the correction that applies in the case of fluid with variable temperature:

$$
f_{w}=f_{w}^{0} \cdot \mathcal{C}_{f}
$$

The coolant friction factor $f_{w}^{0}$ is modeled by means of the Colebrook's correlation ${ }^{20}$ because of its capability to evaluate the rough wall effect and the skin friction correction factor $\mathcal{C}_{f}$ by means of the Petukhov's formula: ${ }^{21}$

$$
\frac{1}{\sqrt{f_{w}^{0}}}=-2 \log \left(0.27 \frac{\varepsilon}{D_{h}}+\frac{2.51}{R e \sqrt{f_{w}^{0}}}\right) \quad \text { and } \quad \mathcal{C}_{f}=\left(\frac{T_{w}}{T}\right)^{-0.6+5.6\left(R e_{w}^{*}\right)^{-0.38}}
$$

where $\varepsilon$ is the wall roughness, $D_{h}$ is the channel hydraulic diameter, $R e_{w}^{*}=R e_{w} \cdot \rho_{w} / \rho, \rho_{w}$ is the fluid density at wall and $R e_{w}$ is the wall Reynolds number (that is, the Reynolds number based on the fluid viscosity at wall). The coolant heat transfer coefficient is modeled according to the Niino's correlation ${ }^{1}$ because it is fitted to supercritical hydrogen flow in rough curved channels with strong wall-coolant temperature difference and 
high Reynolds number, as in the case under investigation. The Niino's semiempirical relation is generally expressed as:

$$
N u=N u^{0} \cdot \mathcal{C}_{\xi} \cdot \mathcal{C}_{x} \cdot \mathcal{C}_{I}
$$

where $N u^{0}$ is the Nusselt number for a non-adiabatic, smooth tube and is a function of the Reynolds number and the Prandtl number. To take into account the effect of variable temperature, these non-dimensional parameters are evaluated at the film temperature, which is the mean value between the fluid and the wall temperature:

$$
N u^{0}=0.062 R e_{f}^{0.7} \operatorname{Pr}_{f}^{0.4}
$$

The term $\mathcal{C}_{\xi}$ is a roughness factor to correct for the surface roughness, $\mathcal{C}_{x}$ is an entrance factor to correct for the heat transfer coefficient in the thermally undeveloped region and $\mathcal{C}_{I}$ is a curvature factor to account for a secondary flow effect induced in a curved channel:

$$
\begin{gathered}
\mathcal{C}_{\xi}=\frac{1+1.5 \operatorname{Pr}^{-1 / 6} \operatorname{Re}^{-1 / 8}(\operatorname{Pr}-1)}{1+1.5 \operatorname{Pr}^{-1 / 6} \operatorname{Re}^{-1 / 8}(\operatorname{Pr} \xi-1)} \xi \\
\mathcal{C}_{x}=1+\left(\frac{s}{D_{h}}\right)^{-0.7}\left(\frac{T_{w}}{T}\right)^{0.1}
\end{gathered}
$$

and

$$
\mathcal{C}_{I}=\left[\operatorname{Re}\left(\frac{R_{h}}{R_{c}}\right)^{2}\right]^{ \pm 0.05}
$$

where $\xi=f_{w}^{0}(R e, \varepsilon) / f_{w}^{0}(R e, \varepsilon=0)$ is the ratio of the rough tube isothermal friction factor to the smooth one, $s$ is the channel-axis abscissa whose origin is the channel inlet, $R_{h}$ is the hydraulic radius of the channel cross section $\left(R_{h}=D_{h} / 4\right), R_{c}$ is the channel radius of curvature and the sign + or - in the exponent of the expression for $\mathcal{C}_{I}$ denotes the concave or convex curvature, respectively.

\section{Coupling: CFD and quasi-2D solver interface}

The quasi-2D model is able to solve the coupled wall and coolant flow evolution once thermal boundary conditions are provided. In particular, the model, suited to rectangular channels, requires thermal boundary conditions at the top and bottom walls. The thermal boundary condition can be one of the following:

- assigned wall temperature;

- assigned heat flux;

- assigned convective heat transfer coefficient and adiabatic wall temperature.

On the outer side of the rectangular channel an adiabatic condition is assumed whereas the hot-gas side thermal boundary condition is obtained by the CFD solver. Of course, in principle, neither temperature nor wall heat flux are known, because they depend on the thermal equilibrium between flows and wall, so a first tentative solution has to be computed. This could be done for instance enforcing a first tentative distribution of wall temperature to the CFD solver, then passing the resulting heat flux to the quasi-2D solver, compare the wall temperature computed by the quasi-2D model with the first tentative value and repeat the CFD computation enforcing the wall temperature computed by the quasi-2D solver iteratively until convergence is reached. Analogously it could be done by enforcing a first tentative heat flux distribution. Although this process could successfully converge, a more efficient way is that of enforcing convective heat transfer coefficient and adiabatic wall temperature. In fact in this case only the heat transfer coefficient has to be adjusted which only weakly depends on wall temperature. Therefore, the convective heat transfer coefficient approach is used to provide the boundary condition to the quasi-2D solver. In particular, the coupling procedure is described by the following steps: 
1. CFD computation of the hot-gas flow, enforcing the wall temperature $\left(T_{w, h g}=700 \mathrm{~K}\right.$ at first iteration, the result provided by quasi-2D computation for the next iterations) as boundary condition and evaluation of the heat transfer coefficient $h_{w, h g}$ from the computed heat flux $q_{w, h g}$, the enforced wall temperature and the adiabatic wall temperature $T_{a w}$ :

$$
h_{w, h g}=\frac{q_{w, h g}}{T_{a w}-T_{w, h g}}
$$

2. quasi-2D computation of the coupled coolant flow and wall thermal evolution using $h_{w, h g}$ values from step 1 and $T_{a w}$; the coupled coolant flow and wall analysis provides a new value of $T_{w, h g}$.

Iterations are made repeating steps $1 \& 2$. The process ends when the difference between the computed values of $T_{w, h g}$ in two consecutive global iterations is smaller than an assigned tolerance. In the present coupling procedure, the adiabatic wall temperature $T_{a w}$, needed to evaluate the convective heat transfer coefficient, is evaluated by means of a CFD numerical simulation where the wall boundary condition is set as adiabatic.

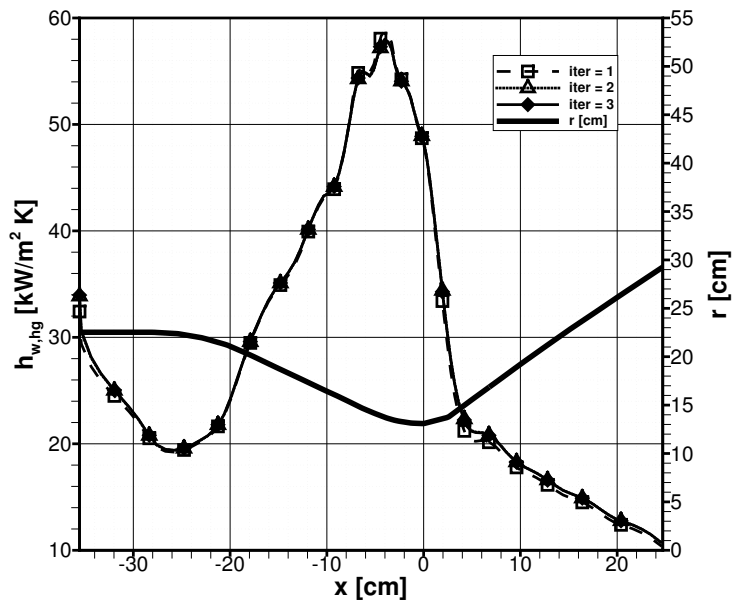

(a) Hot-gas side heat transfer coefficient.

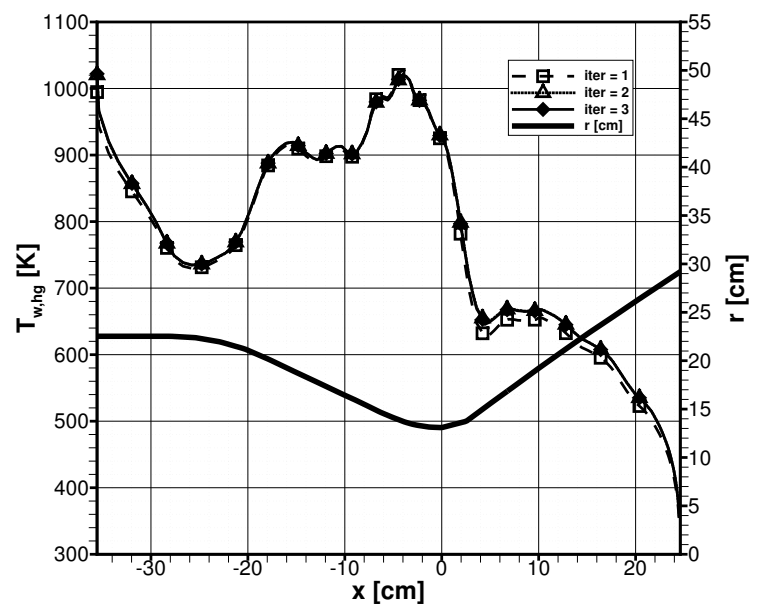

(b) Hot-gas side wall temperature.

Figure 1: Coupling process convergence history.

A typical behavior of the hot-gas side heat transfer coefficient and wall temperature evaluated at different global iterations is presented in Figs. 1 for the test case of interest in this study. It can be noticed that, after the second iteration, the solution is almost overlaid to the previous one. This is clearly noticed in Fig. 1(a), where the maximum difference, located at the abscissa $x=5 \mathrm{~cm}$, between the first two iterations is $4.4 \%$ and vanishes after the second global iteration. Good convergence can be also noticed in Fig. 1(b) where it is shown that wall temperature differences between the first two global iteration are lower than $15 \mathrm{~K}$ along most of the engine and with a maximum difference of $23 \mathrm{~K}$ located at the axial abscissa $x=3.6 \mathrm{~cm}$. Also in this case, after the second global iteration, convergence is fully reached as the solutions do not appreciably change any more. Thus, the convergence history of the hot gas side heat transfer shown in Figs. 1 reveals that the first iteration is able to provide a very good evaluation of the heat transfer coefficient which is only slightly refined by the following coupling steps.

\section{Test case}

For the sake of comparison with experimental data, a coupled hot-gas/wall/coolant analysis is carried out for the SSME Main Combustion Chamber (MCC), whose data relevant to the regenerative cooling circuit and the thrust chamber profile have been published in the open literature. ${ }^{8,22,23}$ The SSME is an oxygen/hydrogen staged-combustion engine which uses the fuel as coolant and presents a throttle range 
between $67 \%$ and $109 \%$ of rated thrust. In particular, the selected data refer to the Standard Throat version of the MCC whose throat diameter is $261.75 \mathrm{~mm}$ with a contraction ratio of 3 and an expansion ratio of 5. The liner is made of NARloy-Z copper alloy and provides the coolant flow path for the MCC. It has 390 milled axial coolant channels that are closed out by an electroforming process which deposits a copper barrier, followed by a nickel Inconel 718 structural closeout over the coolant channels. ${ }^{8,22}$ Coolant moves from the inlet manifold, located at the MCC nozzle exit, to the exit manifold, located at the injector-face section. The channel geometry properties, taken from [22], are reported in Fig. 2.

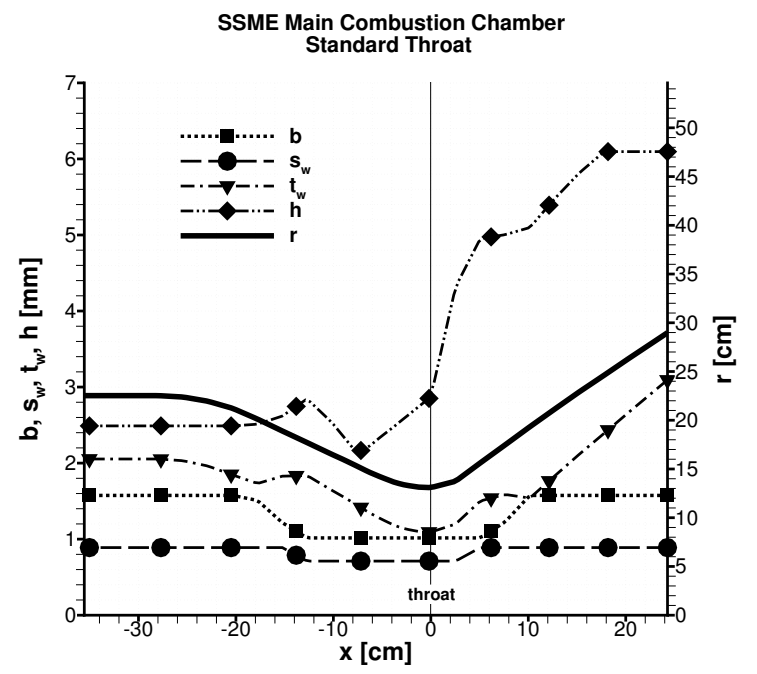

Figure 2: SSME Main Combustion Chamber geometry (data taken from [22]).

Among the available data, the most complete set for the Standard Throat SSME-MCC refers to the Full Power Level operative condition (FPL, $109 \%$ of rated thrust), ${ }^{8,22}$ with chamber pressure $p_{c}=225.87$ bar and oxidizer-to-fuel mixture ratio $O / F=6.0$. The coolant operative conditions at FPL are taken from [8]: overall coolant mass flow rate is $\dot{m}=14.306 \mathrm{~kg} / \mathrm{s}$ (i.e., $36.68 \mathrm{~g} / \mathrm{s}$ for a single channel), inlet temperature is $53.89 \mathrm{~K}$ and inlet pressure is 445.47 bar. Coolant thermodynamic and transport properties used in the fluid solver are taken from [24], while the solid-material thermal conductivity is taken from [25] and refer to that of NARloy-Z copper alloy at $533 \mathrm{~K}: k_{w}=316 \mathrm{~W} / \mathrm{m} \mathrm{K}$.

\section{Results}

In this section, the uncoupled analysis of the hot gas side heat transfer is discussed first to show the capability of the CFD approach to provide a reliable hot gas side heat transfer coefficient. Then, the coupled analysis is discussed comparing the numerical results obtained with experimental data and numerical evaluations retrieved in open literature.

\section{A. Uncoupled analysis}

The hot-gas side domain is discretized by means of a structured axis-symmetric grid shown in Fig. 3. Volumes are clustered toward the wall to solve the boundary layer up to the viscous sublayer. The enforced boundary conditions are shown in Fig. 3: at the subsonic inlet boundary, chamber stagnation pressure and temperature are prescribed together with velocity direction. Stagnation temperature and mixture composition are evaluated as the adiabatic flame temperature and the mixture composition, respectively, under the hypothesis of chemical equilibrium at the chamber pressure and mixture ratio by means of the "Chemical Equilibrium with Applications" (CEA) program. ${ }^{26}$ The outlet section is set as supersonic outflow hence no condition has to be prescribed, the lower boundary is an axis of symmetry and the upper boundary is a no-slip wall. Recalling the coupling process described in Sec. II-C, two hot gas numerical simulations are required for the first step to evaluate the convective heat transfer coefficient by Eq. 9: one for the wall heat flux with a first guess wall temperature and one for the adiabatic wall temperature evaluation. The first 
is obtained by enforcing $T_{w, h g}=700 \mathrm{~K}$ all along the chamber wall, the second is evaluated assuming an adiabatic wall.

Solution grid independence is verified by means of grid convergence analysis in the case with isothermal wall and numerical error is evaluated referring to the Richardson extrapolated solution. ${ }^{27}$ In Table 1 , volumes of the three grids adopted for the analysis are summarized together with the minimum volume dimension at the wall. The $y^{+}$value obtained with the three grid levels along the wall is plotted in Fig. 4(a). The solutions obtained in terms of wall heat flux along the chamber are shown in Fig. 4(b) together with the numerical error relevant to the medium grid size solution. Note that the medium grid size is able to provide a solution with a numerical error lower than $2 \%$. Hence the solution obtained by means of the medium grid size is adopted in the following for the heat transfer analysis.

\begin{tabular}{lcc}
\hline \hline Grid refinement & $\begin{array}{c}\text { Volumes } \\
(\text { axial } \times \text { radial })\end{array}$ & $\Delta y_{\text {min }}$ \\
\hline Coarse grid & $50 \times 45$ & $2 \mu \mathrm{m}$ \\
Medium grid & $100 \times 90$ & $1 \mu \mathrm{m}$ \\
Fine grid & $200 \times 180$ & $0.5 \mu \mathrm{m}$ \\
\hline \hline
\end{tabular}

Table 1: Grid convergence analysis: volumes and minimum volume dimension.

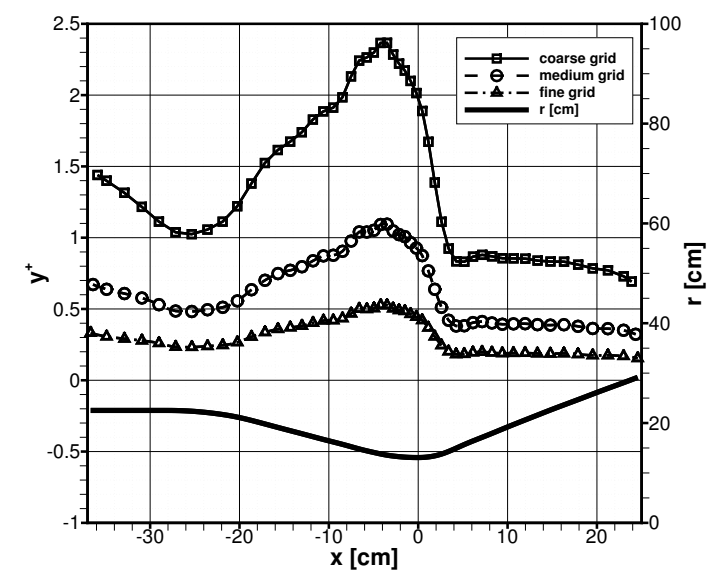

(a) $y^{+}$along the wall.

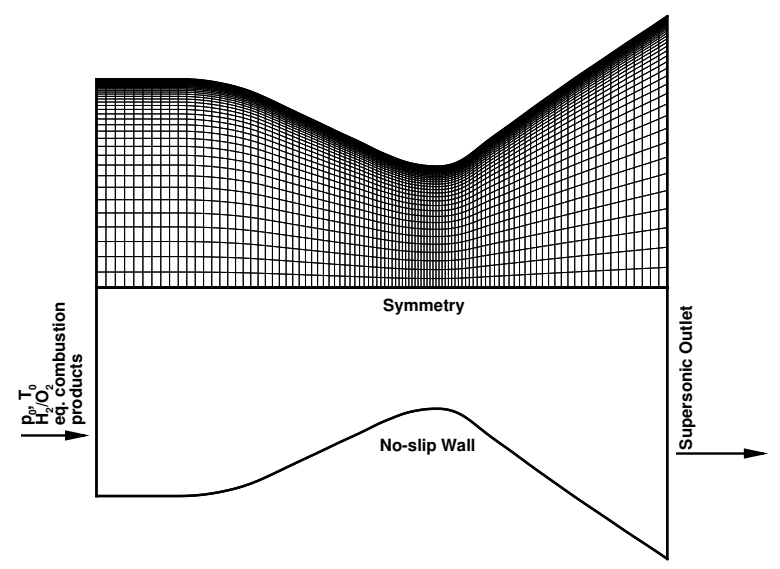

Figure 3: Numerical grid (medium grid size) (top) and boundary conditions (bottom) for the hot gas side domain.

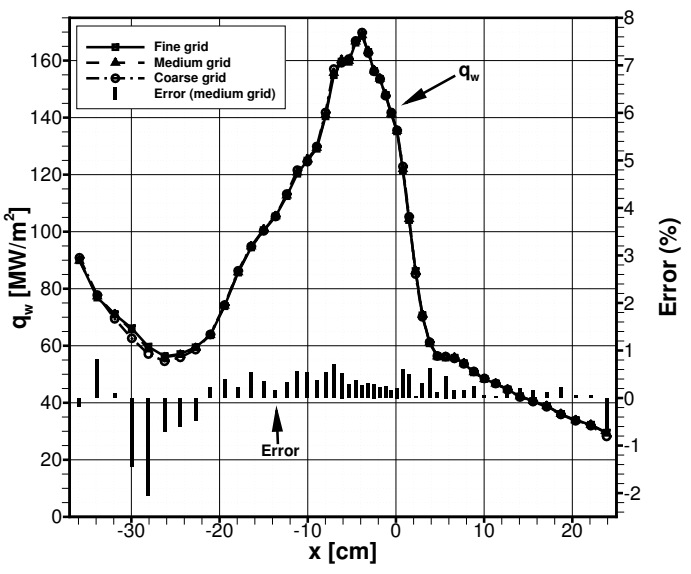

(b) Wall heat flux along the chamber and medium grid size numerical error.

Figure 4: CFD Solution grid independence analysis with three grid levels: isothermal test case $\left(T_{w, h g}=\right.$ $700 \mathrm{~K}$ ).

As described in Sec. III, the heat transfer coupling process sharing the hot-gas side convective heat transfer coefficient together with the adiabatic wall temperature along the thrust chamber ensures a fast convergence within few steps (see Fig. 1(b)). In Fig. 5(a) the hot gas side convective coefficient evaluated 
by means of the CFD numerical simulation is compared to the one measured in the calorimetric test case and scaled as described in [23] and the one obtained by means of the semi-empirical Bartz's equation. ${ }^{28}$

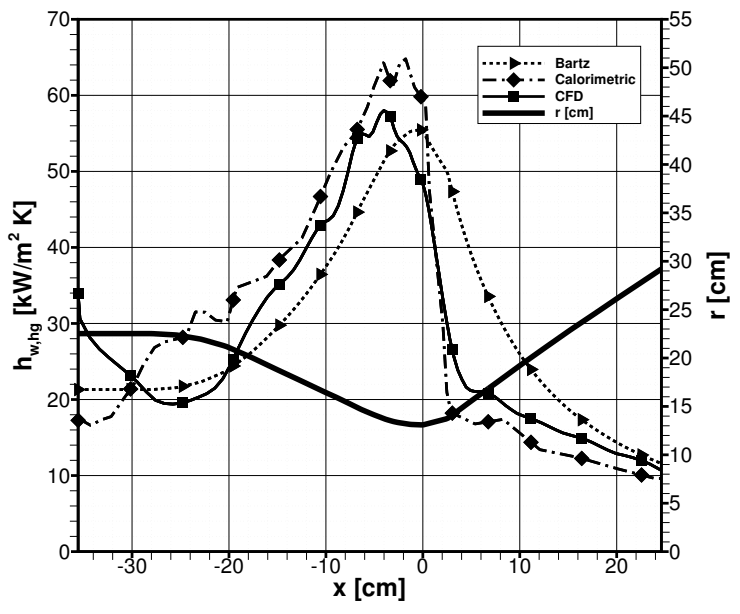

(a) Hot gas side heat transfer coefficient

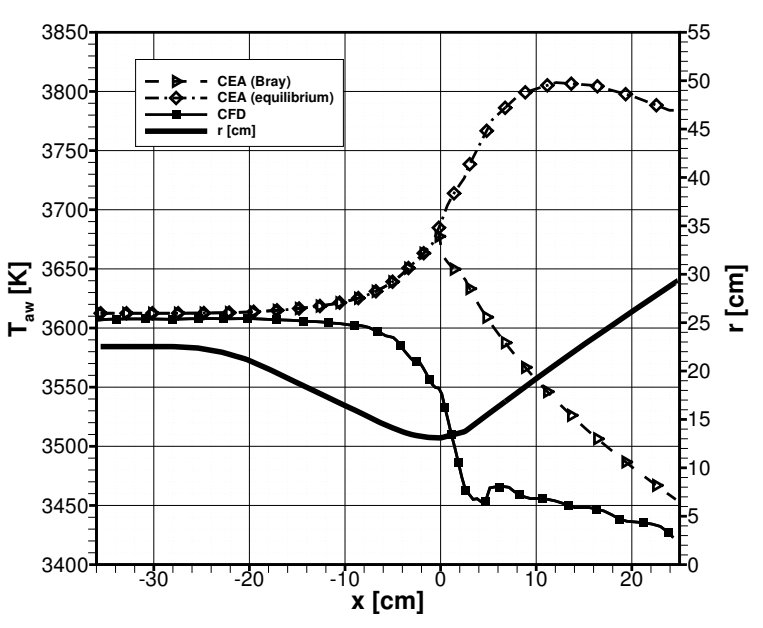

(b) Adiabatic wall temperature

Figure 5: Hot gas side coupling parameters

The CFD numerical solution for the hot gas side convective heat transfer is characterized by an unphysical decreasing trend in first part of the chamber $(x<-25 \mathrm{~cm})$ related to the near injector plate model adopted which does not take into account for the injector effects. Downstream, the coefficient increases as expected reaching its maximum value $\left(58 \mathrm{~kW} /\left(\mathrm{m}^{2} \mathrm{~K}\right)\right)$ at $x=-4 \mathrm{~cm}$ upstream of the throat section. Then the convective coefficient decreases with two different slopes: the steeper across the throat section and the milder downstream in the divergent section. The scaled calorimetric evaluation follows the same trend of the numerical convective coefficient with its maximum value located at the same section upstream of the throat and then decreases with the two different slopes. Nevertheless, they differ in the values: the scaled calorimetric evaluation gives higher convective coefficient in the convergent part whereas reaches lower values in the divergent part. These discrepancies can be attributed to the limits of validity of the scaling laws, which are considered in the estimation of the heat transfer coefficient and the adiabatic wall temperature. ${ }^{23}$ The Bartz prevision does not follow the trend described for the other two methods, showing its maximum value at the throat section and then it mildly decreases toward the divergent part of the nozzle. The convective heat transfer shown in Fig. 5(a) is evaluated from Eq. 9 assuming the adiabatic wall temperature plotted in Fig. 5(b) for each method. For the CFD evaluation, the adiabatic wall temperature along the thrust chamber is evaluated by means of a numerical simulation where adiabatic wall is enforced as boundary condition assuming a frozen composition once the hot gas mixture is injected in the chamber. The scaled calorimetric evaluation is related to the $T_{a w}$ evaluated by means of the CEA program assuming chemical equilibrium all over the chamber as reported in [23]. In the Bartz's equation, $T_{a w}$ is evaluated by means of the CEA program under the Bray hypothesis for which the mixture composition is evaluated at the chemical equilibrium up to the throat section and then it is considered frozen. The three approaches provide similar $T_{a w}$ up to the convergent part of the nozzle and then follow different trends. This is related to their different approaches to evaluate the mixture composition inside the thrust chamber affecting the thermodynamic and transport properties of the flowing gas.

\section{B. Coupled analysis}

The results obtained with the present approach (referred to as "CFD + quasi-2D") are compared with available literature data in Fig. 6. Besides estimations reported in [8] (referred to as "Wang and Luong"), which also include the heat fluxes predicted by Rocketdyne (referred to as "Design Method A"), the present solutions are obtained with two different levels of wall roughness: $0 \mu \mathrm{m}$ (smooth) and $0.23 \mu \mathrm{m}($ rough). The latter value of roughness has been selected in order to have the same pressure drop of [8]. 


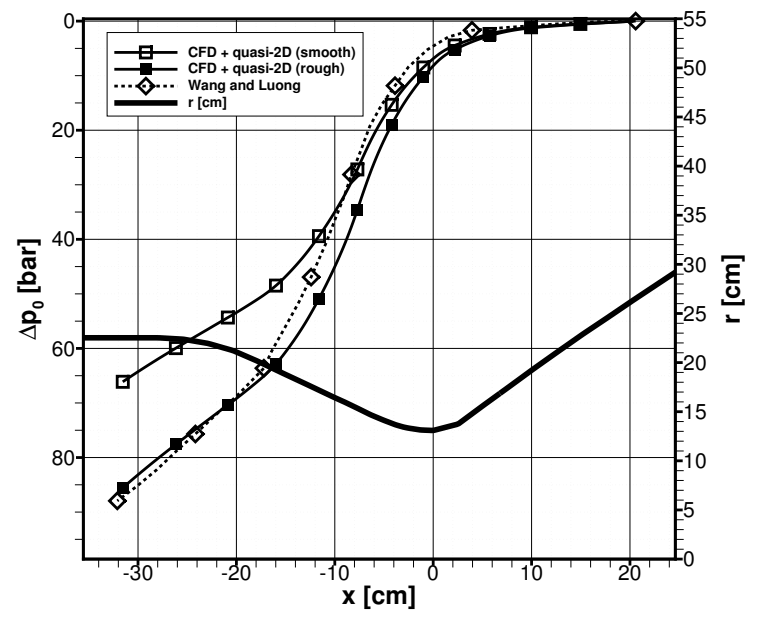

(a) Total pressure loss.

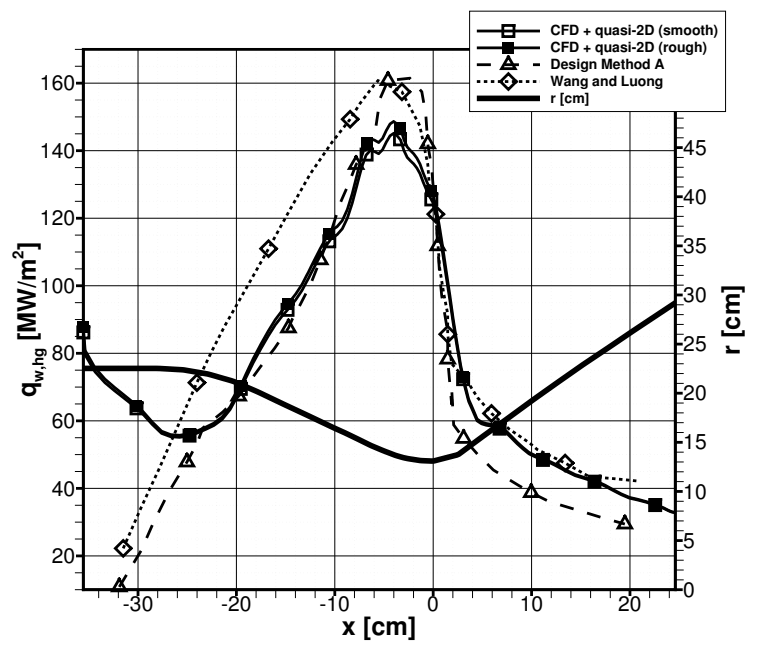

(c) Hot-gas side heat flux.

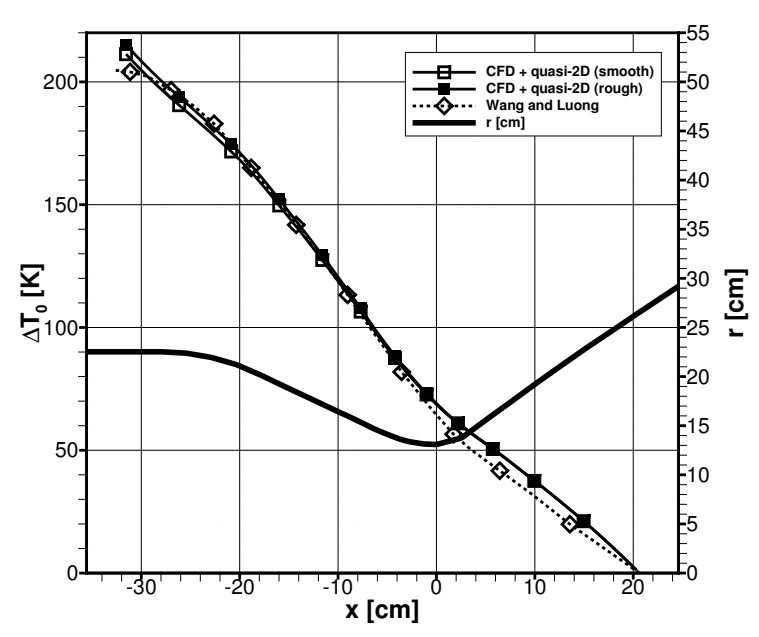

(b) Bulk total temperature increase.

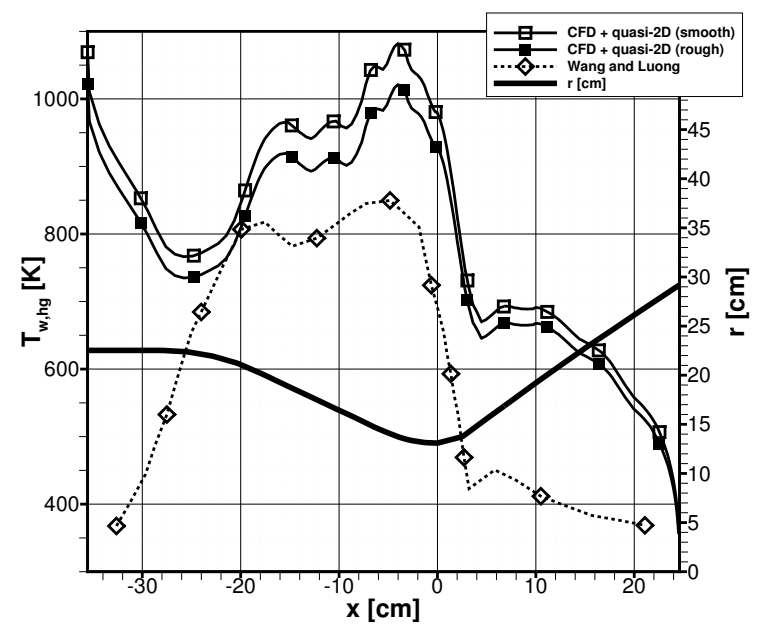

(d) Hot-gas side wall temperature.

Figure 6: SSME Main Combustion Chamber, Standard Throat, FPL operative condition. Comparison of present and literature numerical simulations along the engine axis.

Pressure loss behavior is presented in Fig. 6(a). The pressure loss estimated with the present approach and the hypothesis of smooth wall is 66.1 bar, which is $25 \%$ lower than that of [8] (87.9 bar). Pressure loss discrepancy could be partially explained by the assumption of smooth walls. In fact, with the hypothesis of cooling channel with rough wall, the present coupled approach agrees with results of [8] if the mentioned level of roughness is assumed. The coolant temperature increase is shown in Fig. 6(b), where it can be seen that the selected level of roughness implies mild differences with respect to the smooth case and that the present approach provides similar results with respect to [8]. In fact, differences among curves are always within $10 \mathrm{~K}$. The comparison of hot-gas side heat fluxes (Fig. 6(c)) shows that the present results difference with respect to those presented in [8] is of the order of $10 \%$. In fact, the peak value, which is achieved in the throat region, is $161.5 \mathrm{MW} / \mathrm{m}^{2}$ with Design Method $A$ and $160.8 \mathrm{MW} / \mathrm{m}^{2}$ with the Wang and Luong estimation while it is $148.6 \mathrm{MW} / \mathrm{m}^{2}$ and $145.1 \mathrm{MW} / \mathrm{m}^{2}$ in the present study with rough and smooth wall, respectively. In the other regions of the thrust chamber, the present results are within those presented in [8] all along the chamber but in the barrel section of the engine $(x<-25 \mathrm{~cm})$ where the hot-gas side heat 
transfer is strongly influenced by the near injector plate modeling; in particular, present results are close to the Wang and Luong estimations in the divergent part and close to Design Method $A$ in the convergent part whereas they are unrealistic in the barrel section. This is because of the adopted hot-gas side modeling which does not consider the reacting flow from the injector plate, but a flow of combustion products in chemical equilibrium using a full-inlet approach. ${ }^{15}$ Even larger differences can be seen analyzing the wall temperature behavior (Fig. 6(d)). In particular, the computed hot-gas side wall temperature is much larger than expected, the maximum value, in the throat region, being $1082 \mathrm{~K}$ or $1021 \mathrm{~K}$ for the present model with the hypothesis of smooth or rough wall, respectively, compared to the $850 \mathrm{~K}$ declared in the open literature. $^{8,25}$ However, except the barrel section where differences are expected because of the adopted CFD modeling, the qualitative behavior of $T_{w, h g}$ is similar to that of Wang and Luong: a peak value slightly upstream of the throat, a local minimum at nearly $130 \mathrm{~mm}$ upstream of the throat and a strong variation downstream of the throat, followed by a local maximum. It is worth to notice that the beneficial effect of the wall roughness on the wall temperature implies a reduction of nearly $60 \mathrm{~K}$. Here it is also noted that, since the computed hot-gas side heat transfer coefficient $h_{w, h g}$ and adiabatic wall temperature $T_{a w}$ are able to provide, for a given heat flux $q_{w, h g}$ of $160 \mathrm{MW} / \mathrm{m}^{2}$, a wall temperature $T_{w, h g}$ of $850 \mathrm{~K}$ in the throat region via Eq. (9), the achieved wall temperature overshoot with respect to result of [8] can be addressed to the coolant flow-modeling only. However, besides the nominal maximum wall temperature and heat flux of the SSME MCC, ${ }^{8,22,25}$ experimental evidences based on full-scale hot-fired engines have evidenced that the operating temperature of the hot wall is much higher than expected, up to more than $1030 \mathrm{~K} .{ }^{29,30} \mathrm{This}$ result is very similar to that achieved with the present numerical approach and gives a strong confidence to its validity and accuracy. Of course, the throat-region heat flux seen in Fig. 6(c), which is lower than the nominal one $\mathrm{e}^{8,22}\left(\sim 160 \mathrm{MW} / \mathrm{m}^{2}\right)$, is a direct consequence of the larger hot-gas side wall temperature (Fig. 6(d)), for a given hot-gas side heat transfer coefficient and adiabatic wall Eq. (9).

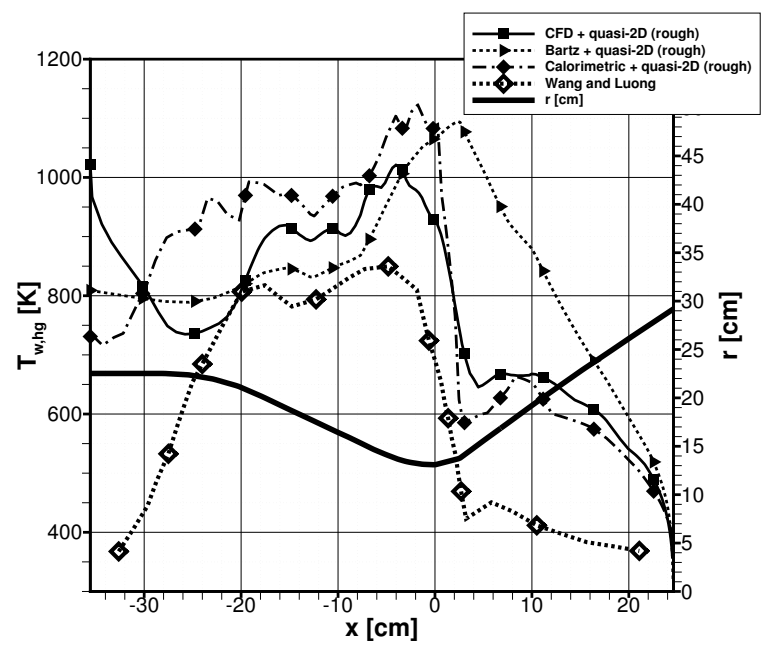

Figure 7: SSME Main Combustion Chamber, Standard Throat, FPL operative condition. Comparison of present and literature numerical solution of hot-gas side wall temperature along the engine axis.

The hot-gas side wall temperature obtained with the different heat transfer coefficients presented in Fig. 5(a) (that are, CFD, Bartz, and Calorimetric solutions) and with the hypothesis of rough wall is presented in Fig. 7 together with that presented in [8]. Both the estimations achieved with the Bartz's correlation and with the scaled heat transfer coefficient from the calorimetric chamber ${ }^{23}$ give a maximum wall temperature in the throat region which is larger than that achieved with the CFD computation. In particular, the larger value of the wall temperature derived from the calorimetric chamber data is due to the larger value of $h_{w, h g}$ (Fig. 5(a)); in this case the peak value in the throat region is $1123 \mathrm{~K}$ and the qualitative behavior of $T_{w, h g}$ is similar to that estimated by Wang and Luong. Furthermore, the estimation achieved with the Bartz's heat transfer coefficient has a peak value of $1094 \mathrm{~K}$ which is located, differently from other predictions, downstream of the throat section. In fact, due to the strongly different behavior of $h_{w, h g}$ predicted with the Bartz's correlation with respect to the others (Fig. 5(a)), the wall temperature is 
far larger in the divergent part of the engine and its general behavior cannot be compared to that of Ref [8].

\section{Conclusion}

A coupled procedure for the simulation of the behavior of the hot-gas/wall/coolant environment in a regeneratively cooled liquid rocket engine, has been set up and demonstrated on the regenerative cooling circuit of an operative rocket engine. The analysis of the SSME MCC has shown a reasonable agreement with published data of coolant pressure drop and temperature gain. Also data concerning the hot-gas side wall temperature are in agreement with experimental measures taken from full-scale hot-fired engines, even if they are in disagreement with the nominal design point. In particular, maximum wall temperature is higher than the nominal value. As a consequence, the maximum computed hot-gas side heat flux results to be lower than the nominal value. Considering the hot-gas side heat transfer coefficient evaluated by the Bartz's model and the scaled data of a calorimetric chamber of the SSME MCC, the wall temperature results to be $100 \mathrm{~K}$ higher than that predicted with the present approach and in case of the Bartz's model the general behavior of the wall temperature is in evident disagreement with the other solutions.

\section{Acknowledgments}

The numerical simulations presented were done at the CASPUR High Performance Computing (HPC) facilities within the "Standard HPC grant" of 2011 and 2012.

\section{References}

${ }^{1}$ Niino, M., Kumakawa, A., Yatsuyanagi, N., and A.Suzuki, "Heat Trasfer Characteristics of Liquid Hydrogen as a Coolant for the L02/LH2 Rocket Thrust Chamber with the Channel Wall Construction," AIAA Paper 1982-1107, June 1982, 18th SAE/ASME Joint Propulsion Conference.

${ }^{2}$ Frohlich, A., Popp, M., Schmidt, G., and Thelemann, D., "Heat Transfer Characteristics of $\mathrm{H}_{2} / \mathrm{O}_{2}$ : Combustion Chambers," AIAA Paper 1993-1826, July 1993, 29th AIAA/ASME/SAE/ASEE Joint Propulsion Conference.

${ }^{3}$ Wadel, M. F., "Comparison of High Aspect Ratio Cooling Channel Designs for a Rocket Combustion Chamber," AIAA Paper 1997-2913, July 1997, 33rd AIAA/ASME/SAE/ASEE Joint Propulsion Conference.

${ }^{4}$ Marchi, C. H., Laroca, F., da Silva, A. F. C., and Hinckel, J. N., "Numerical Solutions of Flows in Rocket Engines with Regenerative Cooling," Numerical Heat Transfer, Part A: Applications, Vol. 45, No. 7, 2004, pp. 699-717.

${ }^{5}$ Naraghi, M. H., Dunn, S., and Coats, D., "A Model for Design and Analysis of Regeneratively Cooled Rocket Engines," AIAA Paper 2004-3852, July 2004, 40th AIAA/ASME/SAE/ASEE Joint Propulsion Conference.

${ }^{6}$ Kirchberger, C., Wagner, R., Kau, H., Soller, S., Martin, P., Bouchez, M., and Bonzom, C., "Prediction and Analysis of Heat Transfer in Small Rocket Chambers," AIAA Paper 2008-1260, July 2008, 44th AIAA/ASME/SAE/ASEE Joint Propulsion Conference.

${ }^{7}$ Pizzarelli, M., Carapellese, S., and Nasuti, F., "A Quasi-2D Model for the Prediction of Wall Temperature of Rocket Engine Cooling Channels," Numerical Heat Transfer, Part A: Applications, Vol. 60, No. 1, July 2011, pp. 1-24.

${ }^{8}$ Wang, T. S. and Luong, V., "Hot-Gas-Side and Coolant-Side Heat Transfer in Liquid Rocket Engine Combustors," Journal of Thermophysics and Heat Transfer, Vol. 8, No. 3, 1994, pp. 524-530.

${ }^{9}$ Liu, Q., Luke, E., Cinnella, P., and Tang, L., "Coupling Heat Transfer and Fluid Flow Solvers for Multidisciplinary Simulations," Journal of Thermophysics and Heat Transfer, Vol. 19, No. 4, 2005, pp. 417-427.

${ }^{10}$ Zhang, H. W., He, Y. L., and Tao, W. Q., "Numerical Study of Film and Regenerative Cooling in a Thrust Chamber at High Pressure," Numerical Heat Transfer, Part A: Applications, Vol. 52, No. 11, 2007, pp. 991-1007.

${ }^{11} \mathrm{Li}$, J. W., Liu, Y., and Qin, L. Z., "Numerical Simulation of Flow and Heat Transfer in Round-to-Rectangular Nozzles," Numerical Heat Transfer, Part A: Applications, Vol. 51, No. 3, 2007, pp. 267-291.

${ }^{12}$ Naraghi, M. H. and Jokhakar, J., "A CFD-RTE Model for Thermal Analysis of regeneratively Cooled Rocket Engines," AIAA Paper 2008-4557, July 2008, 44th AIAA/ASME/SAE/ASEE Joint Propulsion Conference.

${ }^{13}$ Kang, Y. D. and Sun, B., "Numerical Simulation of Liquid Rocket Engine Thrust Chamber Regenerative Cooling," Journal of Thermophysics and Heat Transfer, Vol. 25, No. 1, 2011, pp. 155-164.

${ }^{14}$ Knab, O., Frey, M., Görgen, J., Maeding, C., Quering, K., and Wiedmann, D., "Progress in Combustion and Heat Transfer Modelling in Rocket Thrust Chamber Applied Engineering," AIAA Paper 2009-5477, Aug. 2009, 45th AIAA/ASME/SAE/ASEE Joint Propulsion Conference.

${ }^{15}$ Betti, B., Flow Field and Heat Transfer Analysis of Oxygen/Methane Liquid Rocket Engine Thrust Chambers, Ph.D. thesis, "Sapienza" University of Rome, in press.

${ }^{16}$ Betti, B., Martelli, E., Nasuti, F., and Onofri, M., "Numerical Study of Film Cooling in Oxygen/Methane Thrust Chambers," 4th European Conference for Aerospace Sciences, July 2011.

${ }^{17}$ Betti, B., Martelli, E., and Nasuti, F., "Heat Flux Evaluation in Oxygen/Methane Thrust Chambers by RANS Approach," AIAA Paper 2010-6721, July 2010, 46th AIAA/ASME/SAE/ASEE Joint Propulsion Conference. 
${ }^{18}$ Spalart, P. and Allmaras, S., "A One-equation Turbulence Model for Aerodynamic Flows," La Recherche Aerospatiale 1, 5-23, 1994 .

${ }^{19}$ Kacynski, K., "Thermal Stratification Potential in Rocket Engine Coolant Channels," NASA-TM 4378, May 1992.

${ }^{20}$ Colebrook, C. F., "Turbulent Flow in Pipes with Particular Reference to the Transition Region Between the Smooth and Rough Pipe Flow," J. Inst. Civ. Eng., , No. 11, 1938, pp. 133-156.

${ }^{21}$ Petukhov, B., "Heat Transfer and Friction in Turbulent Pipe Flow with Variable Physical Properties," Advances in Heat Transfer, Vol. 6, 1970, pp. 503-564.

${ }^{22}$ Cook, R. T., Fryk, E. E., and Newell, J. F., "Space Shuttle Main Engine Main Combustion Chamber Life Prediction," NASA CR-168215, 1983, Final Report, Rocketdyne Division Rockwell International, Canoga Park, CA.

${ }^{23}$ Dexter, C. E., Fisher, M. F., Hulka, J. R., Denisov, K. P., Shibanov, A. A., and Agarkov, A. F., "Scaling Techniques for Design, Development, and Test," (in Liquid Rocket Thrust Chambers: Aspects of Modeling, Analysis, and Design) Progress in Astronautics and Aeronautics Series, Vol. 200, 2004, pp. 553-600.

${ }^{24}$ Younglove, B. A., "Thermophysical Properties of Fluids. I. Argon, Ethylene, Parahydrogen, Nitrogen, Nitrogen Trifluoride, and Oxygen," Journal of Physical and Chemical Reference Data, Vol. 11, No. 1, 1982, pp. 1-353.

${ }^{25}$ Esposito, J. J. and Zabora, R. F., "Thrust Chamber Life Prediction. Volume 1: Mechanical and Physical Properties of High Performance Rocket Nozzle Materials," NASA CR-134806, 1975, Final Report Boeing Aerospace Co., Seattle, WA.

${ }^{26} \mathrm{McBride}, \mathrm{B}$. J. and Gordon, S., "Computer Program for Calculation of Complex Chemical Equilibrium Compositions and Applications," NASA Reference Publication 1311, 1994.

${ }^{27}$ Roy, C. J., McWherter-Payne, M. A., and Oberkampf, W. L., "Verification and Validation for Laminar Hypersonic Flowfields, Part 1: Verification," AIAA Journal, Vol. 41, No. 10, October, 2003.

${ }^{28}$ Bartz, D. R., "An Approximate Solution of Compressible Turbulent Boundary-Layer Development and Convective Heat Transfer in Convergent-Divergent Nozzles," Trans. ASME, Vol. 77, No. 8, 1955, pp. 1235-1245.

${ }^{29}$ Sanders, J. and Chen, P., "Degradation of a Main Combustion Chamber Liner on a Space Shuttle Main Engine," Handbook of Case Histories in Failure Analysis, Vol. 1, 1992, pp. 56-60.

${ }^{30}$ Singh, J., Jerman, G., Bhat, B., and Poorman, R., "Microstructural Stability of Wrought, Laser and Electron Beam Glazed NARLOY-Z Alloy at Elevated Temperatures," NASA-TM 108431, Nov. 1993. 